\title{
Time resolved Nomarski interferometery of laser produced plasma plumes
}

\author{
P. Hough ${ }^{\text {a,* }}$, C. McLoughlin ${ }^{\text {a }}$, T.J. Kelly ${ }^{\text {a }}$, S.S. Harilal ${ }^{\text {b }}$, J.P. Mosnier ${ }^{\text {a }}$, J.T. Costello ${ }^{\text {a }}$ \\ ${ }^{a}$ School of Physical Sciences and National Centre for Plasma Science and Technology, Dublin City University, Glasnevin, Dublin 9, Ireland \\ ${ }^{\mathrm{b}}$ Prism Computational Sciences, Inc., 455 Science Drive, Suite 140, Madison, WI 53711, USA
}

\section{A R T I C L E I N F O}

\section{Article history:}

Available online 28 August 2008

\section{Keywords:}

Laser

Plasma

Nomarski

Interferometery

Pulsed laser deposition

Electron density

Plasma scale length

\begin{abstract}
A B S T R A C T
We report results from optical interferometric probing of a laser generated $\mathrm{Zn}$ plasma plume. The experiment was performed in a vacuum and $\mathrm{O}_{2}$ rich environments where the background pressure of $\mathrm{O}_{2}$ was maintained at $1000 \mathrm{~Pa}$ and the results from both regimes are compared. The focus of our work is very much on the early stages in the life of the plasma plume which remains, to date, a largely unexplored area of study, at least in the pulsed laser deposition research domain. It was found that the electron density profile normal to the target is different in the background gas at early times ( $\sim 30 \mathrm{~ns})$ compared to that of the vacuum case. At later times ( $\sim 80 \mathrm{~ns})$ both profiles have a very similar shape. We also observe the formation of a shock wave at the plasma-gas interface shortly after plasma breakdown $(<15 \mathrm{~ns})$.
\end{abstract}

(c) 2008 Elsevier B.V. All rights reserved.

\section{Introduction}

PLD is a well-established method in materials science and has spawned various applications such as thin film deposition with opto-electronic applications [1], bio-compatible materials [2] and growth of nano-structured materials [3]. Considerable research and development has been carried out on the growth and characterisation of $\mathrm{ZnO}$ in particular, due to its excellent electrical, chemical and optical properties [4]. These confer on $\mathrm{ZnO}$ a wide range of potential applications such as UV opto-electronics devices [5], field emitting displays (FEDs) [6] and anti-bacterial surfaces [7]. The fact that $\mathrm{ZnO}$ has a large exciton binding energy of $60 \mathrm{meV}$ and a large bandgap of $3.37 \mathrm{eV}$ offers real promise for obtaining lasing action at room temperature [8].

One area offering great potential for process monitoring and control has been optical characterisation of the laser generated plasma plume used to deposit material onto the substrate. Most optical diagnostic studies of the plasma plume have focussed on late times ( $>100 \mathrm{~ns}$ ) in the lifecycle of the plume as optical emission spectroscopy (OES) [9] works best when most of the continuum emission has subsided. Consequently, there is a paucity of studies on probing moderate temperature plasma plumes at earlier times $(<100 \mathrm{~ns})$. However, this is a vital stage in the

\footnotetext{
* Corresponding author. Tel.: +3531700 7693.

E-mail address: padraighough@gmail.com (P. Hough).
}

lifecycle of the plume as it determines the future evolution and characteristics of the expanding plasma. It is also during this early phase that the primary interaction between the plume and any background gas present takes place [10].

The electron density of a plasma plume is of particular interest as it plays an important role in defining plume energetics, expansion and interaction with ambient gases [11]. A laser interferometer is the ideal tool as it can very accurately extract the spatio-temporal structure of the electron density at early times in the plasma lifecycle [12]. We report here a study of electron density profiles in laser generated $\mathrm{Zn}$ plasma plumes in both vacuum and $\mathrm{O}_{2}$ rich environments. We have chosen the simpler single element $\mathrm{Zn}$ target rather than $\mathrm{ZnO}$ as our objective is to demonstrate the value of the technique as a PLD diagnostic rather than on PLD of ZnO per se.

\section{Experimental details}

A Nd:YAG Surelite III laser working at its fundamental wavelength of $1064 \mathrm{~nm}$ with an energy of $600 \mathrm{~mJ}$ was used to ablate a flat slab of $99.95 \%$ pure $\mathrm{Zn}$ in both vacuum and $\mathrm{O}_{2}$ rich environments. The pulse width of the laser pulse was $6 \mathrm{~ns}$ and the beam was focused to a spot size of $100 \mu \mathrm{m}$ using a $30-\mathrm{cm}$ planoconvex lens which yielded an irradiance in excess of $10^{11} \mathrm{~W} \mathrm{~cm}^{-2}$. A synchronised Surelite I Nd:YAG laser working at its frequency doubled wavelength of $532 \mathrm{~nm}$ and a pulse width of 5 ns provided the light source for the optical laser interferometer used to probe 


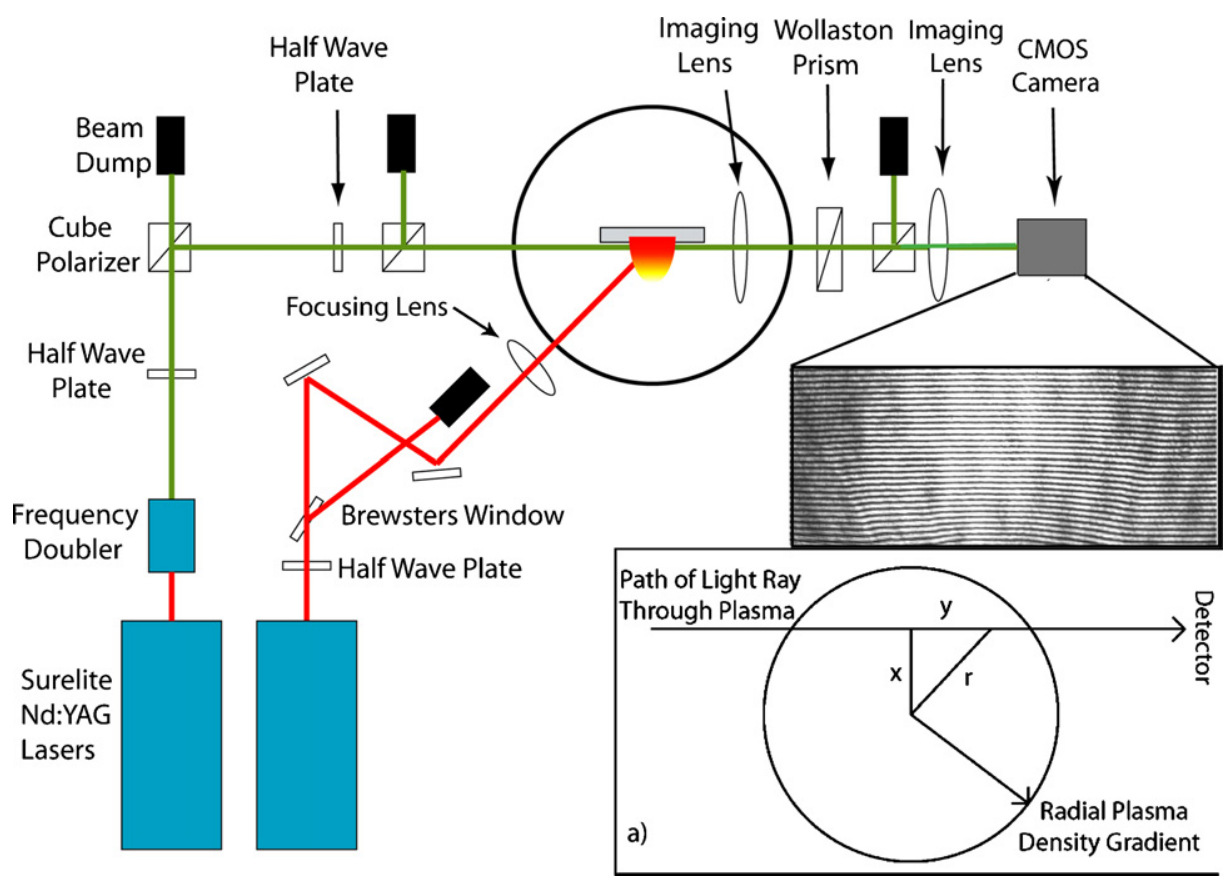

Fig. 1. Schematic setup of experimental setup. Inset (a): definition of plasma coordinate system.

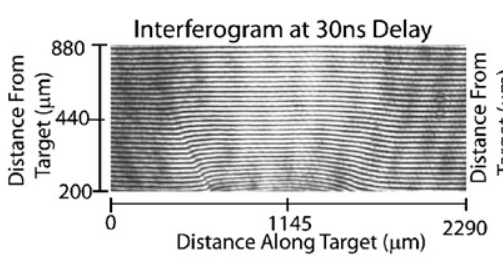

Electron Density at 30ns Delay $\left(\mathrm{cm}^{\wedge} \wedge-3\right)$

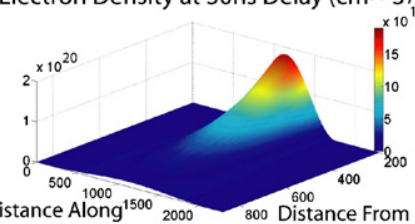

Target $(\mu \mathrm{m})$

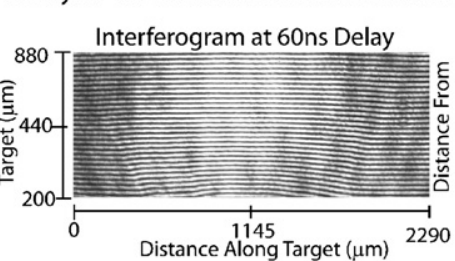

Electron Density at 60 ns Delay $\left(\mathrm{cm}^{\wedge}-3\right)$

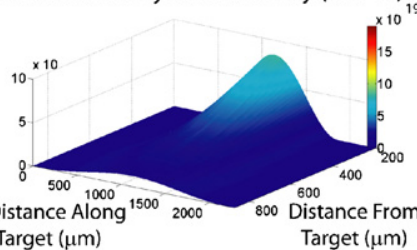

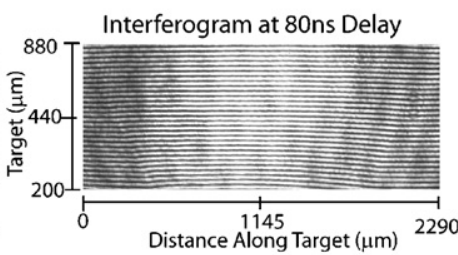

Electron Density at $80 \mathrm{~ns}$ Delay $\left(\mathrm{cm}^{\wedge}-3\right)$

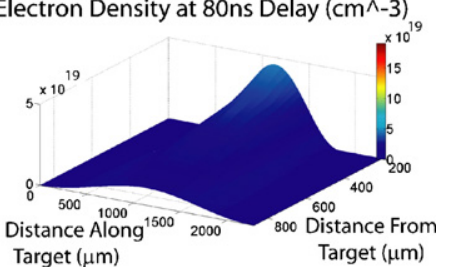

Analysis of Zn Plume in Oxygen Enviroment
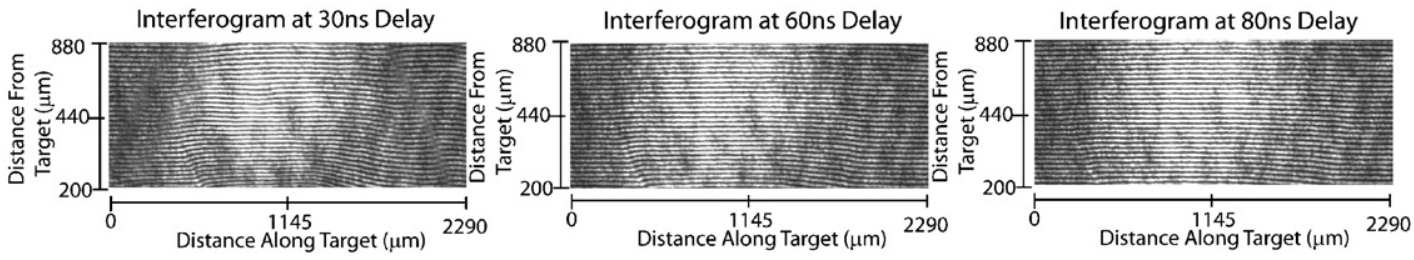

Electron Density at 30ns Delay $\left(\mathrm{cm}^{\wedge} \mathrm{-}-3\right)$

Electron Density at 60ns Delay $\left(\mathrm{cm}^{\wedge}-3\right)$
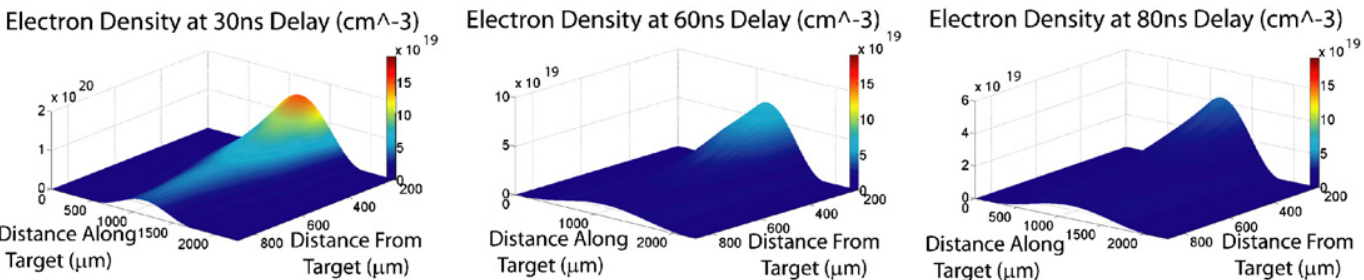

Fig. 2. Comparison of results obtained from optical interferograms taken at time delays of 30,60 and 80 ns for the $\mathrm{Zn}$ plume in vacuum and in 1000 Pa $\mathrm{O}_{2}$ pressure. 
the spatio-temporal profile of the electron density in the plasma plume. A schematic diagram of the experimental setup is shown in Fig. 1.

The interferometer is of the Nomarski polarisation type [13]. The two lasers were synchronised to a CMOS camera (C Cam Ltd., Model: BCi4) using two Stanford DG535 delay generators. A TTL master pulse was sent to trigger the Surelite III laser and create the $\mathrm{Zn}$ plasma. The $532 \mathrm{~nm}$ laser delay was varied to probe the $\mathrm{Zn}$ plasma plume at different times in its life cycle. The camera shutter was left open to integrate for $1 \mu$ s during which time it captured the 5 ns duration interferogram. A narrow bandpass filter centred at $532 \mathrm{~nm}$ rejected broadband optical emission from the plasma plume.

A high precision gas flowmeter was used to introduce $99.999 \%$ pure $\mathrm{O}_{2}$ into the vacuum chamber at a rate of $400 \mathrm{sccm}$ creating a pressure of $1000 \mathrm{~Pa}$. The $\mathrm{Zn}$ target was mounted on a high precision in-vacuum motorised $X-Z$ stage and was moved to reveal a fresh surface after each laser shot.

\section{Results and discussion}

Before discussing the results we outline the basic operation of the interferometer and how it was used to extract electron density maps. The electron density gradient of the laser produced plasma induces a fringe shift in the interferograms which is recorded by a CMOS camera (Fig. 1). The electron density profile, $n_{\mathrm{e}}$, of the laser produced plasma can be calculated for a relative fringe shift using the Abel equation [14]:

$n_{\mathrm{e}} \cong-\lambda_{\mu \mathrm{m}} n_{\mathrm{c}} \int_{r}^{r_{\max }} \frac{\mathrm{d} \Phi}{\mathrm{d} x}\left(x^{2}-r^{2}\right)^{-1 / 2} \mathrm{~d} x$

where $\lambda_{\mu \mathrm{m}}$ is the wavelength of the probe laser in microns, $n_{\mathrm{c}}$ is the critical density of the plasma at the probe wavelength, $\Phi$ is the phase shift and $x$ and $r$ are the plasma coordinates as defined in by inset (a) in Fig. 1. Interferograms were taken at $5 \mathrm{~ns}$ intervals up to a delay of 100 ns after plasma formation. In Fig. 2 we show results of laser interferometery for two different regimes (vacuum and $1000 \mathrm{~Pa} \mathrm{O}_{2}$ ).

Interferograms were analysed using the software package IDEA [15] (interferometric data evaluations algorithm) where we have assumed axial symmetry of the laser generated plasma plume around the normal to the target. This assumption is justified by a study [16] of the plume emission which shows that the distribution is symmetric about an axis normal to the target. In addition we have assumed that only free electrons contribute to the refractive index of the plasma. This assumption is justified by the fact that there are no transitions in $\mathrm{Zn}$ atoms or ions within $\sim \pm 1 \mathrm{~nm}$ of the probe laser beam wavelength [17]. The area of interest in the interferogram selected for analysis $(200 \mu \mathrm{m}$ from target surface) is free from light absorption near the target surface where the electron density is greater than the critical density of the $532 \mathrm{~nm}$ probe laser beam. What is immediately clear from Fig. 2 is that the peak electron density is very high in the early stages of the plasma lifecycle ( $\sim 30 \mathrm{~ns})$ and lies just above $10^{20} \mathrm{~cm}^{-3}$ for both scenarios, albeit the vacuum case is slightly higher ( $20 \%$ higher at $200 \mu \mathrm{m}$ see Fig. 2 (top)). It is also apparent from Fig. 2, that the spatial profile of the electron density of the plumes and their development over time is different for the vacuum and oxygen cases. These two points are emphasised in Fig. 3 where we compare the 1D profile of the electron density normal to the target for vacuum and oxygen environments at time delays of 30 and $80 \mathrm{~ns}$.

In the vacuum case a simple exponential function fits the experimentally observed electron density spatial profile, as the plasma is freely expanding in vacuum. To calculate the plasma density scale length we follow the same convention as Santala et al. [18] which yields a value of $240 \mu \mathrm{m}$ for the vacuum case at a time delay of $30 \mathrm{~ns}$. In the $\mathrm{O}_{2}$ environment the density profile cannot be fitted with a single exponential. In fact a double exponential with a rapidly decaying term (scale length $=125 \mu \mathrm{m}$ ) superimposed on a slowly decaying term (scale length $=1440 \mu \mathrm{m}$ ) works best. We propose that these observations are due to the effects of the strong plasma confinement which can be expected when a plasma expands into a background ambient gas. With an increase in gas pressure, an increase in the frequency of electron and ion collisions with background atoms and molecules takes place [19], which results in increased ionisation, recombination and hence plasma emission [20-23]. In particular Liu et al. [20] and Leboeuf et al. [21,24] have predicted, using 2D particle hydrodynamic plume expansion models, that as a result of the confinement of the plasma, the plasma density is found to remain higher inside the shock wave than in the free expansion case. It is approximately three times higher at $900 \mu \mathrm{m}$ (Fig. 3a) in our study. To make a direct comparison between the profiles we calculate that in vacuum the density drops by a factor of $1 / e$ at a distance of $240 \mu \mathrm{m}$ from the target and it drops by a factor of $2 / e$ at $495 \mu \mathrm{m}$. We obtain $1 / e$ and $2 / e$ values of 310 and $740 \mu \mathrm{m}$ respectively in $\mathrm{O}_{2}$. So we can clearly see that there is a fast decay component and a slow decay component in the $\mathrm{O}_{2}$ case at a time delay of 30 ns. In Fig. 3b however we show the profile of the electron density normal to the target for both vacuum and oxygen ambient atmospheres at a time delay of $80 \mathrm{~ns}$. Although the density in the vacuum case is slightly higher, the main observation here is that both profiles can be fitted using a single exponential function. From these fits we obtain a plasma density scale length of 500 and $410 \mu \mathrm{m}$ for the vacuum and oxygen environments respectively at a time delay of $80 \mathrm{~ns}$. It is clear that by 80 ns the shock wave has dissipated sufficiently for its confinement effect to have largely disappeared in the region close to the target.
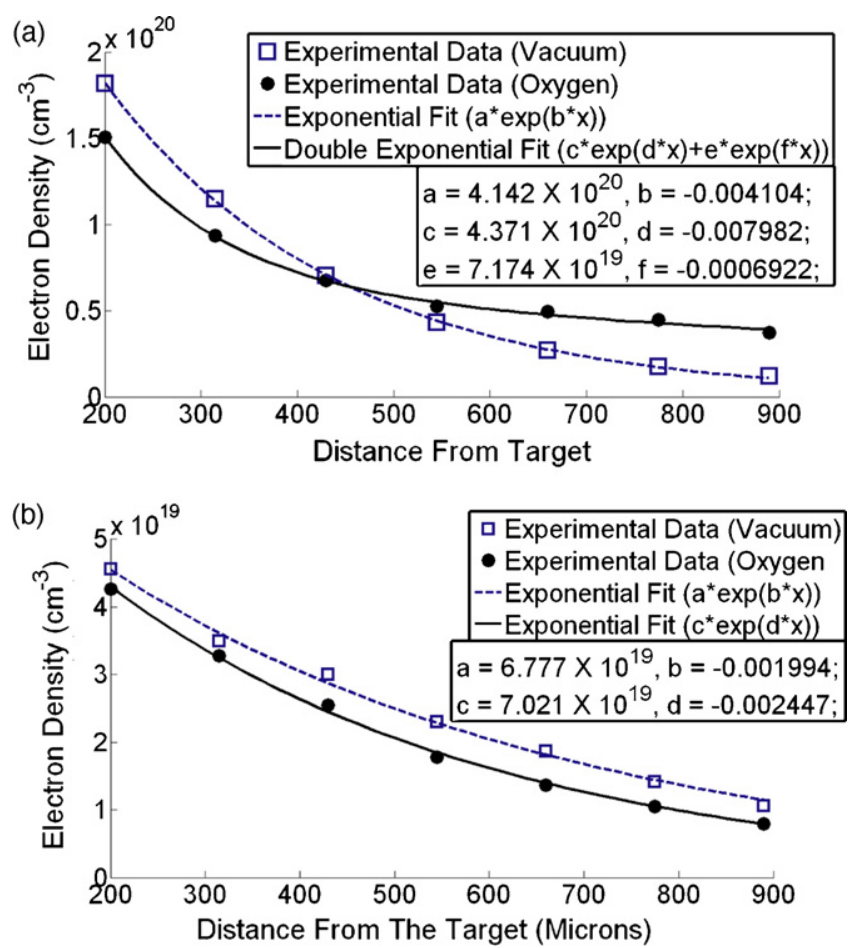

Fig. 3. Comparison of the electron density profile normal to the target for vacuum and oxygen environments at (a) time delay of $30 \mathrm{~ns}$ and (b) time delay of $80 \mathrm{~ns}$. 


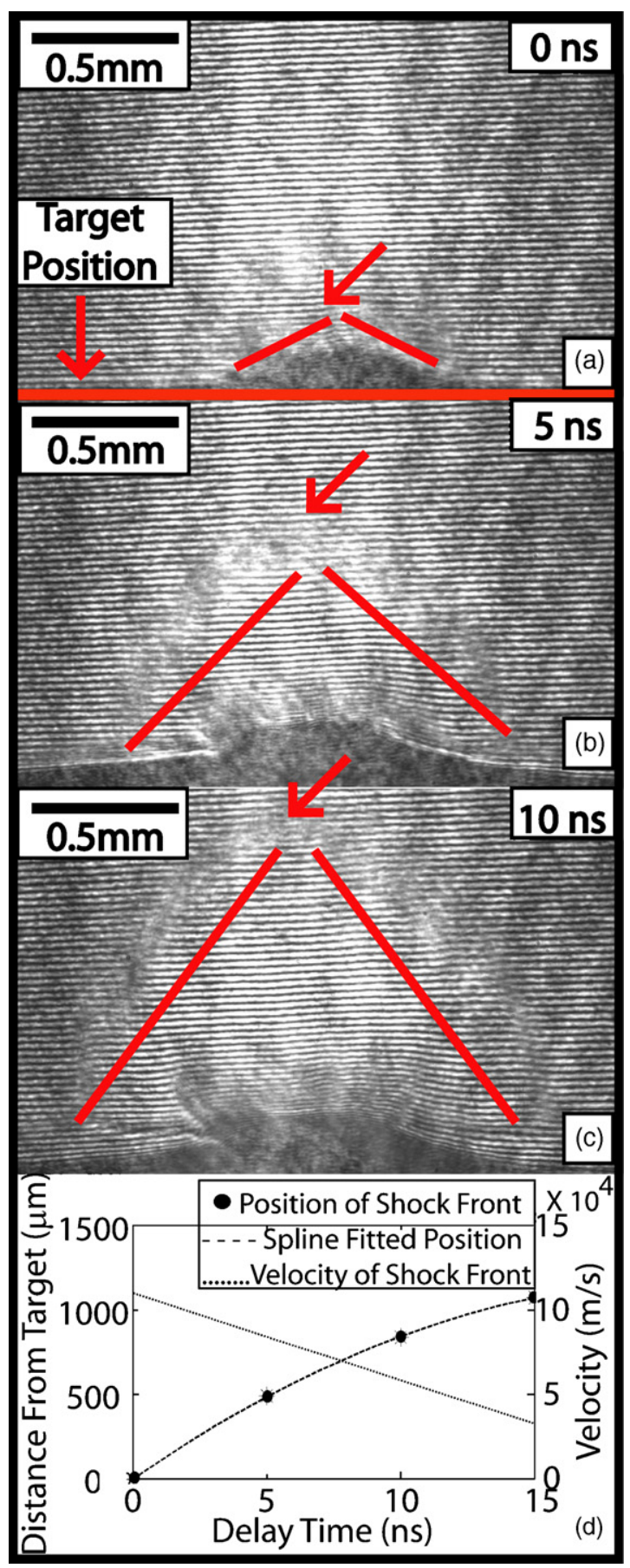

Fig. 4. Comparison of interferograms for (a) $0 \mathrm{~ns}$, (b) $5 \mathrm{~ns}$ and (c) $10 \mathrm{~ns}$ with the shock front highlighted. The arrow indicates the peak (largest distance from target) of the shock front. (d) Plot of the position of the shock front as a function of time with a fitted spline curve along with the resulting velocity.

As the key signature of this plasma confinement is the development and evolution of shock waves as the plasma plume expands in the gaseous environment, we finish with a brief look at their development in our particular case. The shock wave acts as a compressive layer formed at the interface between the plasma plume and the background gas and is observed to expand outward normal to the target over time. It manifests itself in the interferograms as a blurring of the fringes due to the gas compression in the region. This is shown in Fig. 4 where interferograms are compared for time delays of 0,5 and 10 ns.

Although shock waves in gases are well documented in the literature $[20,25]$ these results reveal the creation of a shock wave at the very earliest times of the plume lifecycle ( $<15 \mathrm{~ns}$ ). A sharpening of the shock wave (the so called "snowplow" effect) is observed in the $10 \mathrm{~ns}$ interferogram which has been predicted $[21,26]$ and observed at late times using fast photography [25]. Our observations reveal its formation at very early times and to our knowledge for the first time in optical diagnostics. In Fig. 4d we plot the position of the leading edge of the shock wave as a function of time. We observe that the shock wave starts with quite a large velocity of $1.1 \times 10^{5} \mathrm{~ms}^{-1}$ but over time drag causes a deceleration and the velocity drops to $3.2 \times 10^{4} \mathrm{~ms}^{-1}$, which is a factor of 3 lower than the initial velocity. This deceleration has been observed and predicted previously in the literature $[20,21]$ and is caused by a viscous force as the plume expands into the background gas.

\section{Conclusions}

Two-dimensional spatially and temporally resolved laser interferograms were taken of a $\mathrm{Zn}$ plasma plume revealing the structure and evolution of the electron density profile in vacuum and $1000 \mathrm{~Pa} \mathrm{O}_{2}$ ambient environments. The method can be used to probe the dynamics of laser plumes used for pulsed laser deposition at early stages ( $<100 \mathrm{~ns}$ ) of its lifecycle. This has been a somewhat neglected domain until recently due to the high levels of continuum emission which makes traditional plasma diagnostics based on line-ratio and line-width measurements difficult. However, is of vital importance as the plasma parameters of the plume at this early stage undoubtedly determine the parameters at later times when materials deposition take place. It is also at this early stage that the first interactions between the plume and any background gases takes place. It is found that the spatial profile of the electron density in a gaseous environment deviates from a single exponential function at very early time in the plume lifecycle. A shock wave is observed to be created at very early time delays ( $0-10 \mathrm{~ns}$ ) which snowploughs through the background gas creating a pointed crescent feature at the leading edge. The experiment and results will provide a very useful resource for comparison with any future modelling of laser-generated plasmas in background gases.

\section{Acknowledgements}

This work was sponsored by Science Foundation Ireland under grant number PHY041 and the HEA PRTLI IV programmes of the 2nd National Development Plan programme (NDP2). One of the authors (PH) would like to acknowledge support from the Embark Scholarship Scheme of the Irish Research Council for Science Engineering and Technology. SSH acknowledges funding from the DCU International Visiting Fellows Programme. Work associated with EU COST Action MP0601.

\section{References}

[1] R.K. Gupta, N. Mamidi, K. Ghosh, S.R. Mishra, P.K. Kahol, J. Optoelectr. Adv. Mater 9 (7) (2007) 2211-2216.

[2] J.P. Borrajo, J. Serra, S. Liste, P. González, S. Chiussi, B. León, M. Pérez-Amor, Appl. Surf. Sci. 248 (2005) 355-359.

[3] D. Cattaneo, N. Righetti, C.S. Casari, A.L. Bassi, C.E. Bottani, Appl. Surf. Sci. 253 (2007) 7917-7921.

[4] D. Rajesh, K. Adhikary, S. Ray, Jpn. J. Appl. Phys. 47 (3) (2008) 1501-1506.

[5] F. Li, D.-I. Son, J.-H. Leem, T.W. Kim, W. Dong, Y.-H. Kim, J.. Appl. Phys. 103 (2008) 073511.

[6] R.T. Rajendra Kumar, E. McGlynn, C. McLoughlin, S. Chakrabarti, R.C. Smith, J.D. Carey, J.P. Mosnier, M.O. Henry, Nanotechnology 18 (2007) 215704.

[7] K. Ghule, A.V. Ghule, B.-J. Chen, Y.-C. Ling, Green Chem. 8 (2006) 1034-1041. 
[8] T.-W. Kin, T. Kawazoea, S. Yamazakib, J. Limb, T. Yatsuia, M. Ohtsua, Solid State Commun. 127 (2003) 21-24.

[9] D. Doria, K.D. Kavanagh, J.T. Costello, H. Luna, Meas. Sci. Technol. 17 (2006) 670674.

[10] S.S. Harilal, B. O'Shay, Y. Tao, M.S. Tillack, J. Appl. Phys. 99 (2006) 083303.

[11] A. Bailini, P.M. Ossi, Europhys. Lett. 79 (2007) 35002.

[12] A. Sagisaka, H. Daido, K. Ogura, S. Orimo, Y. Hayashi, M. Nishiuchi, M. Mori, K. Matsukado, A. Fukumi, Z. Li, S. Nakamura, K. Takagaki, H. Hazama, M. Suzuki, T. Utsumi, S.V. Bulanov, T. Esirkepov, Appl. Phys. B 78 (2004) 919-922.

[13] R. Benattar, C. Popovics, R. Sigel, Rev. Sci. Instrum. 50 (1979) 12.

[14] J. Ruiz-Camacho, F.N. Beg, P. Lee, J. Phys. D: Appl. Phys. 40 (2007) 2026-2032.

[15] M. Hipp, J. Woisetschläger, P. Reiterer, T. Neger, Measurement 36 (2004) 53-66

[16] K. Kavanagh, Thesis, submitted for publication.

[17] National Institute of Standards and Technology. http://physics.nist.gov/PhysRefData/ASD/.
[18] M.I.K. Santala, M. Zepf, I. Watts, F.N. Beg, E. Clark, M. Tatarakis, K. Krushelnick, A.E. Dangor, T. McCanny, I. Spencer, R.P. Singhal, K.W.D. Ledingham, S.C. Wilks, A.C. Machacek, J.S. Wark, R. Allott, R.J. Clarke, P.A. Norreys, Phys. Rev. Lett. 84 (2000) 7.

[19] S. Amoruso, J. Schou, J.G. Lunney, EuroPhys. Lett. 76 (3) (2006) 436-442.

[20] C.L. Liu, J.N. Leboeuf, R.F. Wood, D.B. Geobegan, J.M. Donato, K.R. Chen, A.A. Puretzky, Mater. Sci. Eng. B47 (1997) 70-77.

[21] J.N. Leboeuf, K.R. Chen, J.M. Donato, D.B. Geohegan, C.L. Liu, A.A. Puretzky, R.F. Wood, Phys. Plasmas 3 (5) (1996) 2203-2209.

[22] J. Gonzola, F. Vega, C.N. Alfonso, J. Appl. Phys. 77 (12) (1995) 6588-6593.

[23] R.K. Sing, A. Kumar, B.G. Patel, K.P. Subramanian, J. Appl. Phys. 101 (2007) 103301

[24] J.N. Leboeuf, T. Tajima, J.M. Dawson, J. Comp. Phys. 31 (3) (1979) 379-408.

[25] S.S. Harilal, C.V. Bindhu, M.S. Tillack, F. Najmabadi, A.C. Gaeris, J. Phys. D: Appl. Phys. 35 (2002) 2935-2938.

[26] C.V. Budtz-Jorgensen, M.M. Mond, B. Doggett, J.G. Lunney, J. Phys. D: Appl. Phys. 38 (2005) 12. 